\title{
Plasma treatment of bulk niobium surface for superconducting rf cavities: Optimization of the experimental conditions on flat samples
}

\author{
M. Rašković, ${ }^{1}$ J. Upadhyay, ${ }^{1}$ L. Vušković, ${ }^{1, *}$ S. Popović, ${ }^{1}$ A-M. Valente-Feliciano, ${ }^{2}$ and L. Phillips ${ }^{2}$ \\ ${ }^{1}$ Department of Physics, Old Dominion University, Norfolk, Virginia 23529, USA \\ ${ }^{2}$ Thomas Jefferson National Accelerator Facility, Newport News, Virginia 23606, USA
}

(Received 30 November 2009; published 4 November 2010)

\begin{abstract}
Accelerator performance, in particular the average accelerating field and the cavity quality factor, depends on the physical and chemical characteristics of the superconducting radio-frequency (SRF) cavity surface. Plasma based surface modification provides an excellent opportunity to eliminate nonsuperconductive pollutants in the penetration depth region and to remove the mechanically damaged surface layer, which improves the surface roughness. Here we show that the plasma treatment of bulk niobium $(\mathrm{Nb})$ presents an alternative surface preparation method to the commonly used buffered chemical polishing and electropolishing methods. We have optimized the experimental conditions in the microwave glow discharge system and their influence on the $\mathrm{Nb}$ removal rate on flat samples. We have achieved an etching rate of $1.7 \mu \mathrm{m} / \mathrm{min}$ using only $3 \%$ chlorine in the reactive mixture. Combining a fast etching step with a moderate one, we have improved the surface roughness without exposing the sample surface to the environment. We intend to apply the optimized experimental conditions to the preparation of single cell cavities, pursuing the improvement of their rf performance.
\end{abstract}

DOI: 10.1103/PhysRevSTAB.13.112001

PACS numbers: 81.65.Cf, 52.77.Bn, 74.70.Ad

\section{INTRODUCTION}

Plasma etching of niobium $(\mathrm{Nb})$ thin films has been readily used in the production of Josephson tunnel junctions [1]. However, except for our publications [2-4], there are no other reports of plasma treatment of bulk $\mathrm{Nb}$ used for superconducting radio-frequency (SRF) cavities. In discharge plasmas containing chlorine or fluorine radicals, the deformed, contaminated, or oxidized bulk $\mathrm{Nb}$ surface interacts with these radicals to produce volatile $\mathrm{Nb}$ halides. The production of reactive species in the discharge and, consequently, the $\mathrm{Nb}$ removal rate is determined by the input power, the pressure, the temperature, and the radical concentration. The same plasma parameters determine the thickness of the plasma sheath [5]. The plasma sheath is the region in the plasma right above the $\mathrm{Nb}$ surface characterized by a drop in potential perpendicular to the surface, thus providing for anisotropic etching. Positive ions from the plasma, accelerated in the sheath, hit the sample surface where they deposit their energy, recombine, and react. If excessively accelerated, the ions could knock out atoms or atomic conglomerates from the sample surface, thereby increasing the surface roughness. Moreover, they can be implanted into the $\mathrm{Nb}$ surface and increase the level of impurities in the penetration depth region. Therefore, plasma parameters during the plasma etching process have to be balanced to achieve high etching rates without increasing the surface roughness or introducing impurities due to the process.

\footnotetext{
*vuskovic@odu.edu
}

Before a single cell cavity would be etched in plasma discharge, the optimization of experimental conditions was performed on flat $\mathrm{Nb}$ samples. The samples were exposed to a microwave glow discharge system using chlorine $\left(\mathrm{Cl}_{2}\right)$ as a reactive gas. The results of the etching rate as a function of plasma parameters were published in Ref. [2]. We have also presented results of the preliminary surface composition analysis performed by x-ray photoelectron spectroscopy (XPS) [3]. The surface characteristics of the $\mathrm{Nb}$ samples exposed in the repetitively pulsed dc diode system (PLAD), with $\mathrm{BF}_{3}$ as reactive gas, were published elsewhere [4]. The surface roughness is described with two parameters, waviness $\left(W_{a}\right)$ and roughness $\left(R_{a}\right)$. The waviness refers to the tendency of the surface to form largedimension shapes, while the roughness refers to the surface deviation from the large pattern. Our results have shown that the untreated sample had the largest features of all measured samples. The BCP sample had much smaller standard deviation ( $W_{\mathrm{RMS}}$ and $R_{\mathrm{RMS}}$ ) than the untreated sample. All plasma-treated samples had the smallest measured standard deviation, especially with respect to the waviness. In this paper, we discuss the influence of the plasma parameters on the surface properties of the samples exposed in the microwave (MW) glow discharge and the steps taken toward the optimization of plasma etching of bulk Niobium.

\section{EXPERIMENT}

\section{Sample preparation and experimental setup}

In order to determine the influence of the sample preparation history on the surface roughness after exposure to a 
microwave glow discharge, the samples' surface was prepared in one of the following ways. Some samples were left unprocessed (NP), i.e., they were exposed to plasma as received after electron beam cutting, while other were mechanically polished (MP) down to $9 \mu \mathrm{m}$ average roughness. After that, some samples were exposed to 1:1:2 buffered chemical polishing (BCP) mixture for removal of approximately $100 \mu \mathrm{m}$ of the surface. Electropolishing (EP) in 1:9 mixture was performed after BCP for 4 hours removing additional $100 \mu \mathrm{m}$ of the surface. After the surface preparation, we exposed the samples to the reactive gas in the MW cavity discharge system described in Ref. [3]. All samples were treated at the same position in the reactor and at identical plasma conditions.

The diagnostics of the plasma process were performed in situ using optical emission spectroscopy. The surface analysis was performed ex situ, after exposure to plasma. The samples were transported from the reactor to the testing systems using standard protective techniques to prevent the exposure of the samples to the laboratory environment.

The characteristic property of reactive plasma is that any process inside the reactor affects the emitted radiation detected outside the plasma. Thus, the measured radiation reflects the reaction mechanism of the plasma etching process. To correlate the mechanisms that take place in the reactor with measured radiation, one has to perform a detailed analysis that provides real-time information on the process. Optical emission spectroscopy is the most convenient method for the analysis of radiation emitted during the etching process. To use optical emission spectroscopy on the flow as a process monitoring technique, we connected the experimental setup to an Acton SpectraPro500i: Model SP-556 Spectrograph in conjunction with a charged-coupled device (CCD) camera. The spectrometer had a focal length of $0.5 \mathrm{~m}$. It was equipped with a triple grating turret: grating (1) had 3600 grooves per millimeter with a resolution of $0.005 \mathrm{~nm}$ per pixel, grating (2) had 1800 grooves per millimeter with a resolution of $0.02285 \mathrm{~nm}$ per pixel, and grating (3) had 600 groves per millimeter with a resolution of $0.07 \mathrm{~nm}$ per pixel. The CCD camera was an Apogee, Model SPH5-Hamamatsu S70301007, Back-Illum with a pixel array of $1024 \times 122$ and a pixel size of 24 microns. We obtained the line shapes and band intensities, needed for evaluation of the gas and electron temperatures and electron density, by integrating over 10 to 1000 pulses. All spectral measurements were performed side-on with respect to the direction of the flow. Small windows were constructed on the side of the cavity to allow these diagnostic measurements. We made an absolute calibration of the system using the Spectra-Physics Quartz Tungsten Halogen Lamp as the blackbody source.

The ex situ surface diagnostics consisted of post treatment measurements of surface roughness, and chemical composition in the surface layers. We measured the surface roughness using a commercial tapping mode atomic force microscope (AFM) (Digital Instruments: Nanoscope IV). Silicon tips with tip size of $10 \mathrm{~nm}$ were used. The scan sizes were $20 \mu \mathrm{m} \times 20 \mu \mathrm{m}, 50 \mu \mathrm{m} \times 50 \mu \mathrm{m}$, and $100 \mu \mathrm{m} \times 100 \mu \mathrm{m}$, whenever possible. The limiting factor for the Nanoscope IV was the maximum vertical range of $6.5 \mu \mathrm{m}$. The typical measurement of surface roughness is expressed as the root mean square (RMS) roughness,

$$
\mathrm{RMS}=\sqrt{\frac{1}{n} \sum_{i, j}\left(h_{i, j}-\bar{h}\right)^{2}},
$$

where $n$ is the total number of taping points, $i$ is the number of taping points in the $x$ direction, $j$ is the number of taping points in the $y$ direction, $h_{i, j}$ is the height reading for point $(i, j)$, and $\bar{h}$ is the average height reading for the whole scan. The RMS depends on the scan size and instrument resolution. Therefore, it is not recommended to compare the RMS from measurements performed on different instruments and with different scan sizes. To go around this problem and to be able to see the real influence of the plasma etching process on surface roughness, we performed the same size scans of a referenced region before and after exposure to plasma. These results are compared in this paper.

Surface topology and point composition were investigated by using a JEOL 6060LV scanning electron microscope (SEM) with energy dispersive $\mathrm{x}$-ray spectroscopy (EDS) capability. The $\mathrm{Nb}$ samples were investigated in high vacuum mode with scanning resolution of $5.0 \mathrm{~nm}$ and a magnification between 30 and 300000 times. The accelerating voltage for a EDS was $15 \mathrm{kV}$. The covered spectral range contains sensitive $\mathrm{x}$-ray lines of $\mathrm{Nb}, \mathrm{Cl}, \mathrm{O}$, and possible impurities line.

A more detailed composition analysis, including composition of oxide layer formed at the surface of $\mathrm{Nb}$ samples after exposure to air, was performed using Kratos ultra-X-ray photoelectron spectrometer (XPS). This XPS has a monochromatic $\mathrm{Al} K_{\alpha}$ x-ray source with $15 \mu \mathrm{m}$ spatial resolution. XPS has lower a spatial resolution than SEM therefore survey spectra represents average composition over large sample's area and cannot be used for point composition analysis. The sampling depth of the used $\mathrm{x}$-ray source was $10 \mathrm{~nm}$ maximum.

\section{RESULTS AND DISCUSSION}

\section{A. Influence of plasma parameters on etching rate and surface roughness}

Plasma etching of $\mathrm{Nb}$ in the $\mathrm{Ar} / \mathrm{Cl}_{2}$ microwave discharge is mostly chemical in nature, which means that the etching rate is primarily determined by the concentration of reactive species in the plasma. The dependence of the etching rate on the initial concentration of molecular chlorine $\mathrm{Cl}_{2}$, holding other plasma parameters constant, is presented in Fig. 1. Etching rates were obtained ex situ, by 


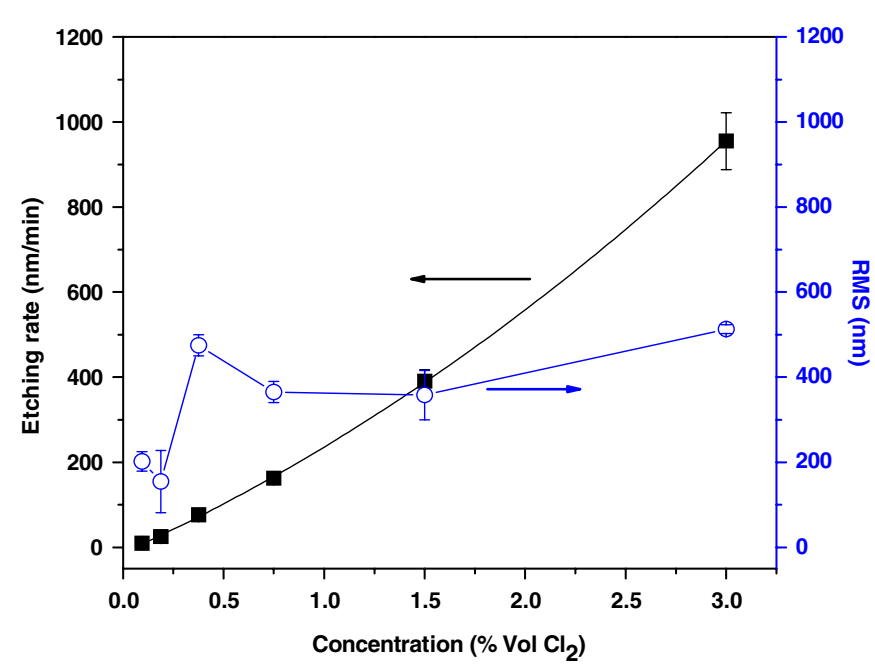

FIG. 1. Etching rate and surface RMS dependence on $\mathrm{Cl}_{2}$ concentration. Experimental conditions: total gas flow $196 \mathrm{sccm}$, pressure in reaction chamber $340 \mathrm{mTorr}$, and input power density $1.40 \mathrm{~W} / \mathrm{cm}^{3}$.

measuring the reduction in mass of the samples after treatment. The initial volume concentration of $\mathrm{Cl}_{2}$ was varied from $0.1 \%$ to $3 \%$. By increasing the initial $\mathrm{Cl}_{2}$ concentration, the etching rate increased steadily with the power of 1.32 , approximately. The surface RMS of the same samples has displayed quite a different behavior, as shown in Fig. 1. The scan RMS size was $20 \mu \mathrm{m} \times 20 \mu \mathrm{m}$. The surface roughness increased by a factor of 2 at low initial concentrations (0.1 to $0.3 \%)$. At higher concentration (0.3 to $3 \%$ ) the surface roughness remained constant, within the statistical error. Since the etching rate at low concentration did not exceed $0.1 \mu \mathrm{m} / \mathrm{min}$, one observes that the reduction of the surface roughness would be achieved to the expense of excessive reduction of etching rates. In most of the present work, the operating concentration was between

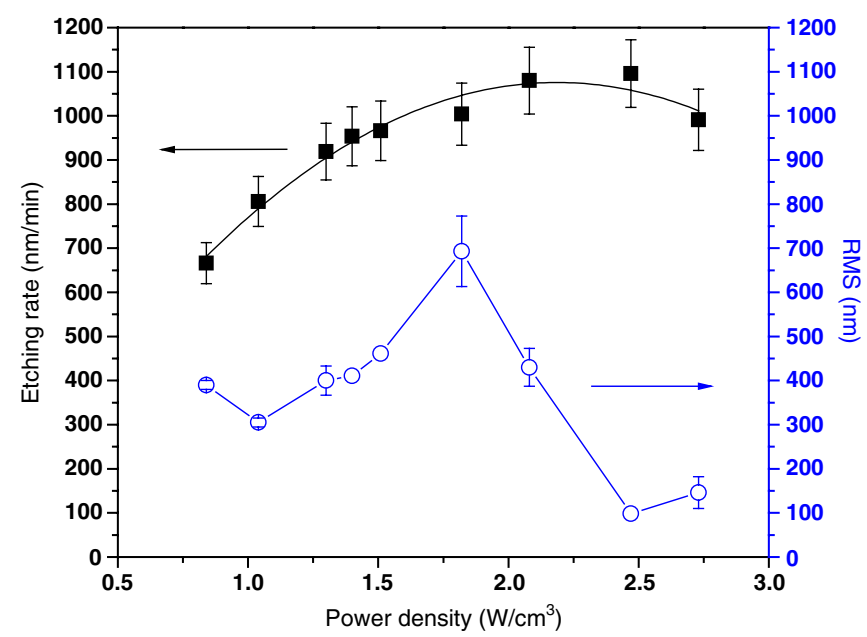

FIG. 2. Etching rate and surface RMS dependence on input power density. Experimental conditions: $3 \%$ of $\mathrm{Cl}_{2}$ in reactive gas mixture and pressure in reaction chamber 340 mTorr.
$2 \%$ and $3 \%$, where we can conclude that the surface roughness does not depend significantly on the concentration of $\mathrm{Cl}_{2}$. The constancy of RMS at higher concentration can be attributed to the kinetics of the chlorine dissociation process, which saturates on the low power density [2] and at relatively low initial concentration of $\mathrm{Cl}_{2}, 3 \%$ of $\mathrm{Cl}_{2}$ in the reaction mixture, that was kept constant while varying all other discharge parameters.

The etching rate depends less strongly on the input power density than on the concentration of $\mathrm{Cl}_{2}$, as shown in Fig. 2. Conversely, the RMS dependence on the input power density shows a peak for medium power densities. Also, we are pointing out that the scan size for the set of measurements presented in Fig. 2 was only $10 \mu \mathrm{m} \times$ $10 \mu \mathrm{m}$ due to instrument limitations. The maximum $z$ range of used atomic force microscope was $6 \mu \mathrm{m}$. When the surface roughness was increased at higher etching rates, it was not possible to obtain $50 \mu \mathrm{m} \times 50 \mu \mathrm{m}$ scans without reaching the instrument $z$-range limit.

$\mathrm{The} \mathrm{Nb}$ etching rate depends much stronger on the pressure in the reaction chamber than on the power density, as shown on Fig. 3. The surface roughness, measured by RMS, is following the same dependence up to 1 Torr, approximately, and drops at higher pressure.

Reasons for this behavior could be found in the increase of plasma density with the increase of pressure and power. The plasma sheath is proportional to the plasma density and therefore, if we increase the power or the pressure, we increase the energy of ions that are hitting the surface. This contributes to an increase of the surface roughness. It has been suggested that this is the best surface quality one could obtain by operating at elevated pressures, but our results do not completely agree with this proposition, as shown in Fig. 3. However, the limited pressure range used in the present work does not allow a definite conclusion.

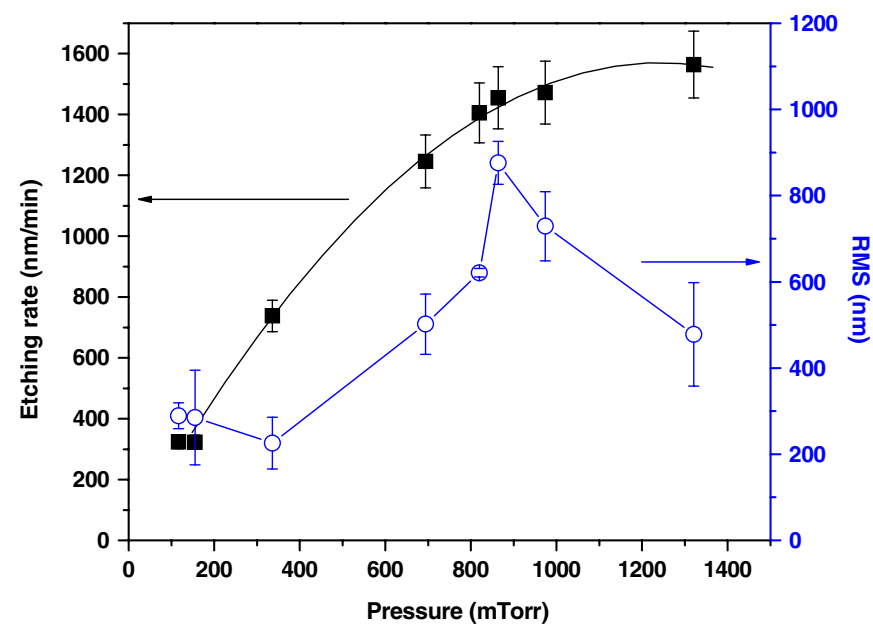

FIG. 3. Etching rate and surface RMS dependence on total gas pressure. Experimental conditions: $3 \%$ of $\mathrm{Cl}_{2}$ in reactive gas mixture, total gas flow $196 \mathrm{sccm}$, and input power density of $1.40 \mathrm{~W} / \mathrm{cm}^{3}$. 
The error bars in Figs. 1-3 represent statistical error obtained through series of measurements of etching rate and RMS under the same experimental conditions. The statistical error for etching rate is $7 \%$, while the statistical errors for RMS vary with the number of scans performed on each sample. Experimental points in all three figures are connected with line as a visual guide, i.e., no fitting procedure is involved.

\section{B. Optical emission spectroscopy}

During the etching process, we performed extensive spectral radiation measurements from plasma layers above the samples. Further, we used optical emission spectroscopy of $\mathrm{Ar} \mathrm{I}, \mathrm{Cl} \mathrm{I}, \mathrm{Nb}$ I lines and $\mathrm{Cl}_{2}$ molecular continuum.

In order to follow thoroughly the assumption of common excitation temperature, we have chosen several sets of spectral lines for each gas in the mixture. The lines had to be recorded in the same CCD camera frame. Further, the lines from the first cascade (labeled with subscript 1 or 2 in Table I) have to be observable, but also the line from the second cascade (labeled with subscript 0 in Table I) has to be of unsaturated intensity. Further, the whole observed column of plasma had to be optically thin for the radiation from these lines. The selected lines for Ar that satisfy the above criteria are listed in Table I.

Taking into account that the excitation temperature is a statistical parameter only, we have inspected its behavior using three separate sets of spectral lines over the full range of power density. The common excitation temperature for all four sets could then serve as a parameter that could characterize the discharge, serve as the indicator for the process, and establish the link from the raw observables to the simulation. Measured excitation temperatures as a function of power density are given in Fig. 4. Results show a fairly constant behavior of all data sets within the statistical error of $15 \%$. Since the accuracy of the data used for the analysis is of the same order, we conclude that the excitation temperature can be used as a common parameter for all data sets that satisfy the conditions of observability and optical thickness that were taken within the same camera frame.

TABLE I. Data of argon line sets.

\begin{tabular}{lllcccc}
\hline \hline Set & & $\begin{array}{c}\Delta E_{i \rightarrow 0} \\
(\mathrm{eV})\end{array}$ & $\begin{array}{c}A \\
\left(10^{6} \mathrm{~s}^{-1}\right)\end{array}$ & $\begin{array}{c}g_{k} \\
(\mathrm{eV})\end{array}$ \\
\hline A & 0 & 800.6156 & & 4.9 & 5 & 13.1718 \\
& 1 & 789.1075 & 1.571 & 0.95 & 5 & \\
& 2 & 805.3308 & 1.539 & 0.86 & 3 & \\
B & 0 & 763.5105 & & 24.5 & 5 & 13.1718 \\
& 1 & 743.5368 & 1.667 & 0.9 & 5 & \\
& 2 & 789.1075 & 1.5708 & 0.95 & 5 & \\
$\mathrm{C}$ & 0 & 840.8209 & & 22.3 & 5 & 13.3022 \\
& 1 & 860.5776 & 1.4403 & 1.04 & 5 & \\
& 2 & 879.9087 & 1.4087 & 0.46 & 3 & \\
\hline \hline
\end{tabular}

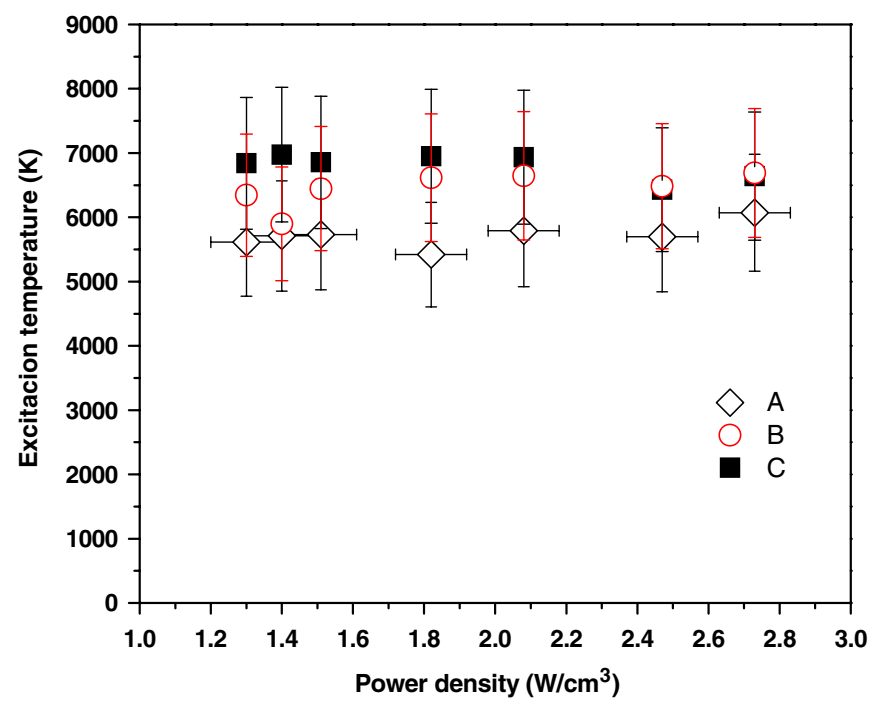

FIG. 4. Excitation temperature in the absence of Nb sample as a function of power density. Error bars reflect the statistical error of the individual sets of data and the uncertainty in local power density. Sets A, B, and C correspond to Table I. Experimental conditions: pressure 1 Torr and the gas mixture $\mathrm{Ar} / \mathrm{Cl}_{2}$ ratio 97:3.

The correlation between the excitation temperature and the etching process is well manifested by the jump at about 3 min after the introduction of $\mathrm{Cl}_{2}$ into the system, as shown in Fig. 5. This event coincides with the increase of the $\mathrm{Nb}$ I and decrease of the $\mathrm{Cl} I$ line intensities, which are shown in Fig. 6. Nb I lines become prominent only after about $3 \mathrm{~min}$ from the start. At the same time, the intensities of atomic $\mathrm{Cl}$ lines drop indicating a delayed reaction which produces volatile $\mathrm{Nb}$ chlorides. After a sharp jump in

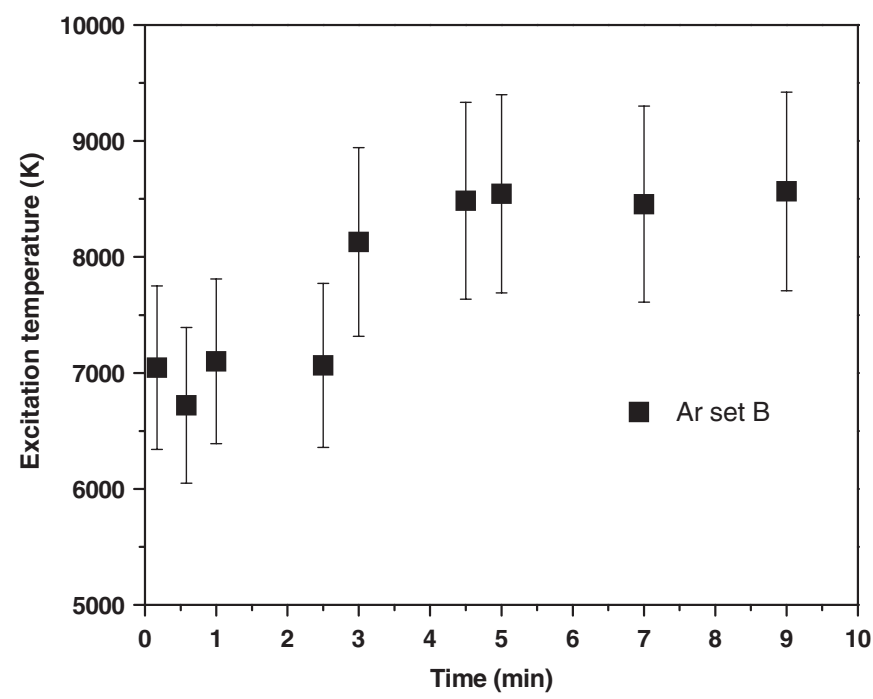

FIG. 5. Excitation temperature as a function of time. Data are taken during the etching process. Set B corresponds to Table I. 


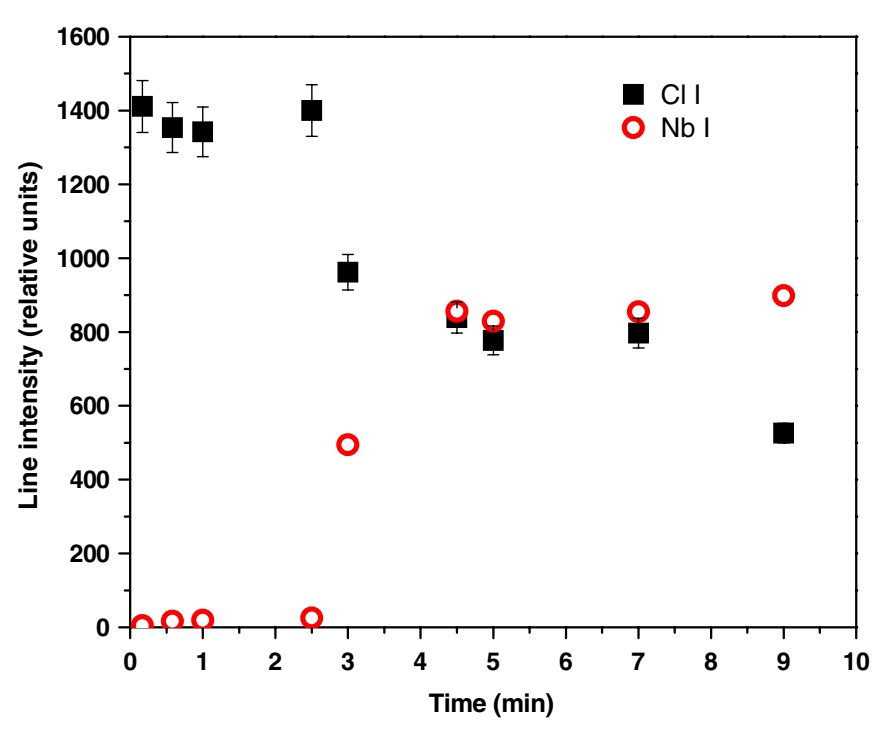

FIG. 6. Intensity of chlorine and niobium lines as a function of time. Data are taken during the etching process. The behavior is typical for all lines. Statistical errors are indicated.

intensity, the $\mathrm{Nb}$ I lines saturate and remain steady during the etching process. While this presents a direct evidence of $\mathrm{Nb}$ presence in the system, the time lag of the process is rather long. The transport processes in the plasma, the equilibration of the electronegative discharge, and the chemical kinetics of the volatile products are certainly contributing to the time delay, but on a time scale that is orders of magnitude lower than observed. The time delay of 3 min rather indicates a surface-breaking process indicative of the initial removal of a protective layer of adsorbed impurities, before the bulk $\mathrm{Nb}$ oxide layer is exposed to the plasma.

\section{XPS surface analysis}

XPS survey spectra have shown only the characteristic lines of $\mathrm{Nb}, \mathrm{O}$, and $\mathrm{C}$. The source of carbon lines was environmental carbon deposited on the surface of samples during handling and transportation. No chlorine lines were found. High-resolution spectra of $\mathrm{Nb}$, are showing $3 d_{5 / 2}$ and $3 d_{3 / 2}$ doublet, separated by $2.75 \mathrm{eV}$ [6]. This $\mathrm{Nb}$ doublet is highly sensitive to the oxide state of $\mathrm{Nb}$. The peak at $201 \mathrm{eV}$ corresponds to metallic $\mathrm{Nb}$ that is the $\mathrm{Nb}_{\mathrm{o}}$ oxidation state. The most intense peak at $207 \mathrm{eV}$ corresponds to $\mathrm{Nb}_{2} \mathrm{O}_{5}$ containing $\mathrm{Nb}^{5+}$ oxidation state. Between these two peaks are located unresolved peaks of $\mathrm{Nb}$ suboxides which oxidation states are $\mathrm{Nb}^{1+}, \mathrm{Nb}^{2+}$, $\mathrm{Nb}^{3+}$, and $\mathrm{Nb}^{4+}$. In both spectra, before and after exposure to the discharge, $\mathrm{Nb}^{5+}$ is the most dominant oxidation state. However, the amount of suboxides is smaller after plasma exposure.

Experiments with $\mathrm{Nb}$ samples in pure Ar plasma show that the discharge removes residues of organic solvents left on the surface during sample preparation. We expected a similar result for the sample exposed to $\mathrm{Ar} / \mathrm{Cl}_{2}$ discharge.
Thus, we show $\mathrm{O} 1 \mathrm{~s}$ line spectra in the Figs. 7 and 8 . According to Ref. [6], the high-energy side wing contains unresolved peaks of $\mathrm{Nb}$ hydroxides and carbonyl group from solvent residues. The wing is significantly smaller after exposure to the discharge. The most intense peak is one that can be associated with $\mathrm{Nb}$ oxides. Its surface increases after exposure to the discharge. We analyzed the observed $\mathrm{O} 1 s$ spectrum from the untreated $\mathrm{Nb}$ sample by a standard deconvolution procedure $[7,8]$. The nonlinear background is assumed and subtracted. The remaining spectrum was normalized to the maximum intensity, located at $530.0 \mathrm{eV}$. By fitting the low energy wing of the normalized $\mathrm{O} 1 \mathrm{~s}$ spectrum, we determined that the best fit was a Voigt profile with $81 \%$ Gaussian and 19\% Lorentzian. The large contribution of the Gaussian component is typical for transition metal oxides. The influence of the instrumental profile is minimal, since we used the monochromatic Al $K \alpha$ line with the full width at half maximum (FWHM) of $0.035 \mathrm{eV}$.

We show in Fig. 7 the result of the $\mathrm{O} 1 s$ line for an untreated sample. The observed spectrum could be fitted with two lines, both containing the same combination of Lorentzian and Gaussian profile. The stronger line was centered at $530.0 \mathrm{eV}$ with the FWHM of $1.24 \mathrm{eV}$. Its position and profile indicates the $\mathrm{Nb}_{2} \mathrm{O}_{5}$ origin. The weaker line was centered at $531.9 \mathrm{eV}$ with $\mathrm{FWHM}$ equal $2.28 \mathrm{eV}$. Based on these two parameters, we suggest that the origin of the second line is unlikely to be from the carbonyl or hydroxyl contribution, but rather from $\mathrm{Nb}$ suboxides. The contribution of the first peak is $70.3 \%$ and the second peak $29.7 \%$.

The oxygen $1 s$ spectrum of the plasma-treated sample is shown in Fig. 8. It differs from the untreated sample in the following: (a) The chemical shift of both lines is reduced and the position of the stronger line is at $529.7 \mathrm{eV}, 0.3 \mathrm{eV}$

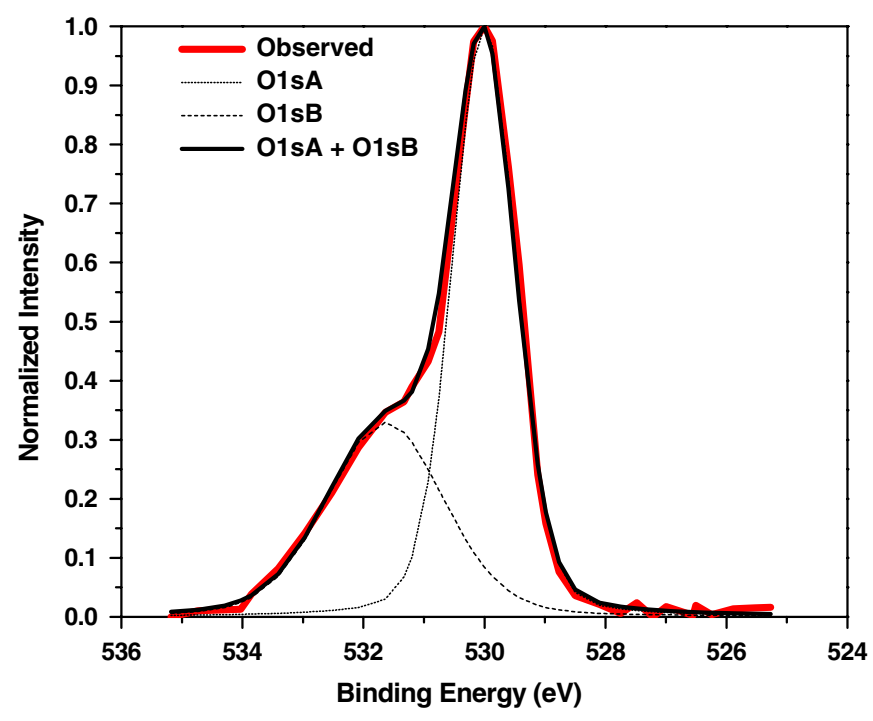

FIG. 7. Normalized XPS spectrum of oxygen $1 s$ for an untreated $\mathrm{Nb}$ sample. 


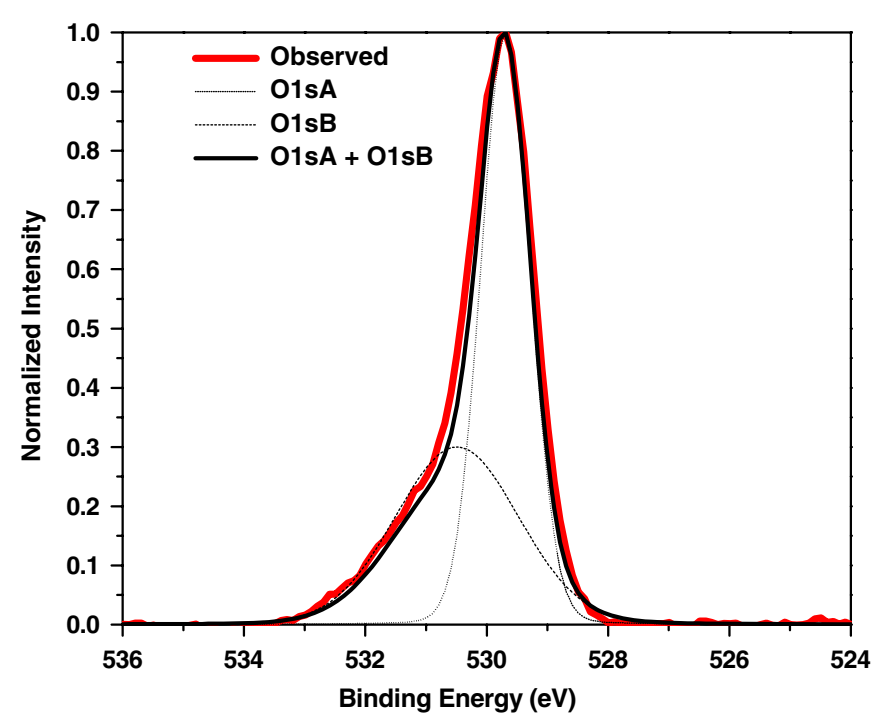

FIG. 8. Normalized XPS spectrum of oxygen $1 s$ for the $\mathrm{Nb}$ sample after $\mathrm{Ar} / \mathrm{Cl}_{2}$ plasma treatment. The same sample is used in Fig. 7 (before treatment) and Fig. 8 (after treatment).

less than for the untreated sample. The position of the weaker line is shifted even more-by about $1.4 \mathrm{eV}$ to be at $530.5 \mathrm{eV}$. (b) The line that originates in $\mathrm{Nb}_{2} \mathrm{O}_{5}$ is narrower for the plasma-treated sample with FWHM equal to $0.96 \mathrm{eV}$, but the second line remains almost unchanged with FWHM at $2.4 \mathrm{eV}$. (c) The Lorentzian contribution drops by a factor of $4 \%$ to $5 \%$, which is observed by increased sharpness of both lines. (d) The relative contribution of the $\mathrm{Nb}_{2} \mathrm{O}_{5}$ line has drops to $65 \%$. A drop of $5 \%$ is within the statistical error.

All this suggests major changes occurring on the molecular level. The small number of exposed samples does not allow us to draw a definite conclusion and further study is planned.

The XPS results also show that, under experimental conditions for the optimal removal rate, no impurities were induced into the sample surface. The chlorine $2 s$ line at $271 \mathrm{eV}$ is absent from the XPS spectrum of plasmatreated samples. The analysis of the surface was also performed on a scanning electronic microscope (SEM). It showed no chlorine lines or any other impurities, except oxygen. An auxiliary analysis performed on the yellow colored deposit from effluent gases in the cold region of the reaction chamber, which was transported to the SEM facility without exposure to air, showed the presence of $\mathrm{Nb}$ and $\mathrm{Cl}$ in a proportion corresponding to $\mathrm{NbCl}_{5}$.

\section{Tri-step plasma etching process}

Taking into account the influence of the plasma parameters on the surface roughness, a tri-step plasma etching process was designed. In the first step, a pure Ar discharge was produced under a total pressure of 500 mTorr and a power density of $2.08 \mathrm{~W} / \mathrm{cm}^{3}$. During the 30 minutes of etching, no $\mathrm{Nb}$ was removed from the surface (etching rate

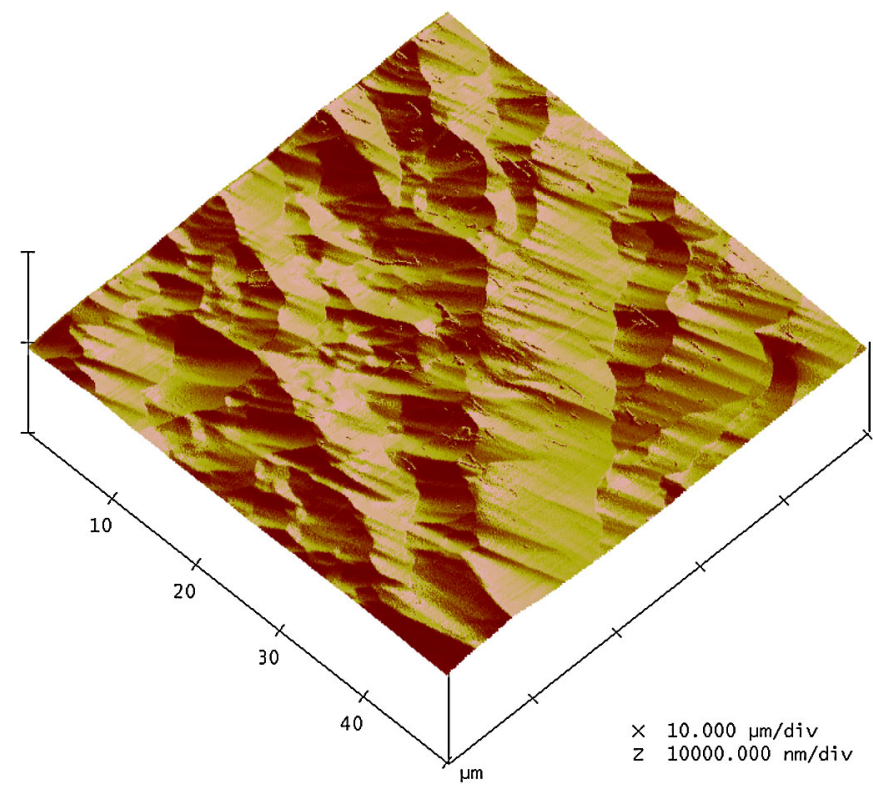

FIG. 9. AFM scan $(50 \mu \mathrm{m} \times 50 \mu \mathrm{m})$ of $\mathrm{BCP}$ prepared surface.

is $0 \mathrm{~nm} / \mathrm{min}$ as shown before [4]) but all physic-sorbed gases and organic residues were removed. Starting with a clean surface, fast plasma etching was performed in the second step. A $3 \mathrm{vol} \% \mathrm{Cl}_{2}$ in $\mathrm{Ar}$ is used as etching gas to remove material as necessary for cavity production. The exposure time was $120 \mathrm{~min}$ under a total pressure of $550 \mathrm{mTorr}$ and input power density of $2.08 \mathrm{~W} / \mathrm{cm}^{3}$. The etching rate in this step was $1 \mu \mathrm{m} / \mathrm{min}$ and approximately $120 \mu \mathrm{m}$ of surface was removed during 2 hours of exposure. The third step was designed to remove $\mathrm{Nb}$ from the surface under conditions more favorable for surface smoothening. $1.5 \mathrm{vol} \% \mathrm{Cl}_{2}$ in $\mathrm{Ar}$ was used under a total pressure of $1250 \mathrm{mTorr}$ and input power density $1.4 \mathrm{~W} / \mathrm{cm}^{3}$. Etching rate was $0.5 \mu \mathrm{m} / \mathrm{min}$. Approximately $100 \mu \mathrm{m}$ of surface was removed during 4 hours of exposure.

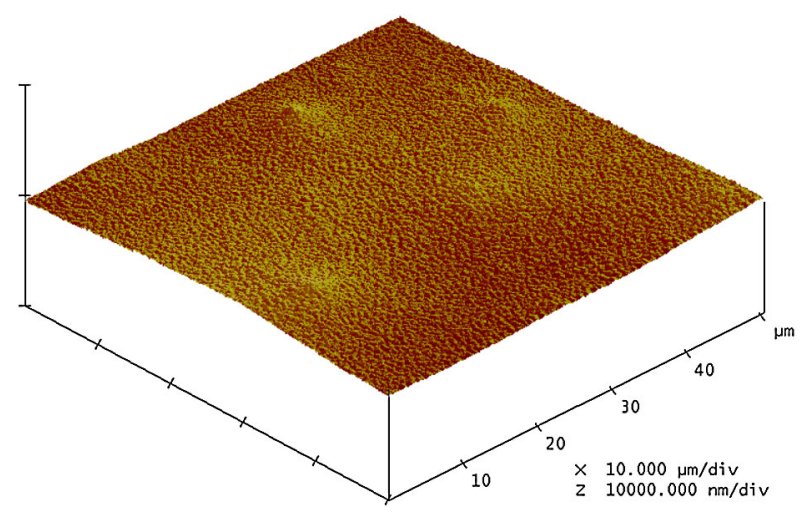

FIG. 10. AFM scan $(50 \mu \mathrm{m} \times 50 \mu \mathrm{m})$ of surface prepared by plasma etching. 
TABLE II. RMS measurement dependence on surface preparation history.

\begin{tabular}{lccc}
\hline \hline $\begin{array}{l}\text { Surface } \\
\text { history }\end{array}$ & $\begin{array}{c}\text { Scan size } \\
(\mu \mathrm{m} \times \mu \mathrm{m})\end{array}$ & $\begin{array}{l}\text { Plasma } \\
\text { etching }\end{array}$ & $\begin{array}{l}\text { RMS } \\
(\mathrm{nm})\end{array}$ \\
\hline $\mathrm{NP}$ & $50 \times 50$ & Before & 254 \\
& & After & 231 \\
$\mathrm{MP}$ & $20 \times 20$ & Before & 758 \\
& & After & 637 \\
$\mathrm{BCP}$ & $50 \times 50$ & Before & 286 \\
& & After & 215 \\
$\mathrm{EP}$ & $50 \times 50$ & Before & 133 \\
& & After & 134 \\
\hline \hline
\end{tabular}

Figures 9 and 10 present the same surface before and after exposure to plasma etching. The surface presented on Fig. 9 is prepared by BCP so the polycrystalline structure is prominent. The plasma etching has eliminated the surface features (Fig. 10). A scanning electron microscope (SEM) was used to observe and understand the nature of the peaks present on the surface after plasma etching.

Because of the element sensitivity of the plasma etching process, the surface preparation history plays an important role in determining the final surface quality. The comparison of RMS measurements before and after the plasma etching process depending on the surface preparation history is presented in Table II. Note that the mechanically polished surface was too rough for a $50 \mu \mathrm{m} \times 50 \mu \mathrm{m}$ scan before plasma processing, therefore $20 \mu \mathrm{m} \times 20 \mu \mathrm{m}$ scan results are presented in this table. From Table II, one can see that plasma etching improves the surface roughness if the starting surface was prepared by mechanical polishing or BCP. The RMS does not change if the surface was prepared by EP. Therefore we can say that plasma etching produces surfaces of lower or comparable roughness with respect to other $\mathrm{Nb}$ surface preparation techniques.

\section{CONCLUSION}

$\mathrm{Nb}$ samples were exposed to the microwave glow discharge in a $\mathrm{Cl}_{2} / \mathrm{Ar}$ mixture in order to establish plasma etching as a viable process for bulk $\mathrm{Nb}$ surface preparation. We were focused on achieving etching rates at a comparable level to wet etching techniques, without introducing process related impurities on the $\mathrm{Nb}$ surface. We also intended to achieve the surface roughness comparable with the currently used wet etching processes.

We have shown that the etching rates of bulk $\mathrm{Nb}$ as high as $1.7 \pm 0.2 \mu \mathrm{m} / \mathrm{min}$ can be achieved in a microwave glow discharge using $\mathrm{Cl}_{2}$ as the reactive gas. The $\mathrm{Nb}$ etching rate depends on the $\mathrm{Cl}_{2}$ concentration and the discharge parameters, such as input power density and pressure in reaction chamber. The surface composition analyses show that no impurities have been introduced into $\mathrm{Nb}$ during the microwave discharge treatment.
To optimize the surface roughness, we have developed a three-step process that gives results comparable to wet processes, producing surfaces of satisfying roughness. The three-step process includes a cleaning step, a fast removal step, and a smoothening step. Further research will be devoted to apply the same experimental conditions to curved surfaces whose shape is closer to the cavity surface. Emission spectroscopy results combined with measured etching rates suggest that the $\mathrm{Nb}$ etching mechanism of MW glow discharge in the $\mathrm{Ar} / \mathrm{Cl}_{2}$ is more a chemical etching process than a physical sputtering one.

A modified experimental setup that includes a single cell cavity has been built. It will be used to determine the influence of the plasma treatment on $\mathrm{rf}$ performance of cavities. The microwave discharge treatment of $\mathrm{Nb}$ surfaces is a convenient, low-cost, and less hazardous alternative to the presently used liquid acid etching techniques.

\section{ACKNOWLEDGMENTS}

This work is supported by the NSF/DOE collaborative effort through the Office of High Energy Physics, Office of Science, Department of Energy under Grant No. DEFG02-05ER41396. M. Rašković and J. Upadhyay acknowledge the financial support from the Accelerator Division, Thomas Jefferson National Accelerator Facility. We thank the Surface Characterization Lab, College of William and Mary Applied Research Center, and the ODU Applied Research Center for the use of various diagnostics equipments. This paper was authored by the Jefferson Science Associates, LLC under U.S. DOE Contract No. DE-AC05-06OR23177.

[1] J. N. Sasserath and J. Vivalda, J. Vac. Sci. Technol. A 8, 3914 (1990).

[2] M. Rašković, J. Upadhyay, L. Vušković, S. Popović, L. Phillips, and A.-M. Valente-Feliciano, J. Vac. Sci. Technol. A 27, 301 (2009).

[3] M. Rašković, L. Vušković, S. Popović, L. Phillips, and A. M. Valente-Feliciano, 13th International Workshop on RF Superconductivity, Beijing, 2007, pp. 323-326, TUP73 [http://accelconf.web.cern.ch/AccelConf/srf2007/ PAPERS/TUP73.pdf].

[4] M. Rašković, L. Vušković, S. Popović, L. Phillips, A.-M. Valente-Feliciano, S. B. Radovanov, and L. Godet, Nucl. Instrum. Methods Phys. Res., Sect. A 569, 663 (2006).

[5] M. Liberman and A. Lichtenberg, Principles of Plasma Discharges and Material Processing (Wiley, New Jersey, 2005).

[6] A. Dacca, G. Gemme, L. Mattera, and R. Parodi, Appl. Surf. Sci. 126, 219 (1998).

[7] D. A. Shirley, Phys. Rev. B 5, 4709 (1972).

[8] E. E. Whiting, J. Quant. Spectrosc. Radiat. Transfer 8, 1379 (1968). 